\title{
AN ALGORITHM FOR DESIGNING FEEDBACK STABILIZERS OF NONLINEAR POLYNOMIAL SYSTEMS.
}

\author{
Stelios Kotsios \\ University of Athens, Faculty of Economics, Department of Mathematics and Com- \\ puter Science. Pesmazoglou 8, 10559 Athens, Greece
}

\begin{abstract}
The aim of this paper is to present a symbolic computational algorithm that will allow us to deal with the feedback stabilization problem for continuous nonlinear polynomial systems. The overall approach is based on a methodology that checks the positivity of a given polynomial.
\end{abstract}

Keywords: Nonlinear Systems, Computational Algebraic Methods, Positivity, Sum of Squares, Feedback Stabilization.

\section{Introduction}

One of the major purposes of control theory is the analysis and the design of feedback control systems. A great number of both analytical and computational methods have also been adopted for continuous and discrete systems, [5], 14].

In this paper we examine the feedback stabilization problem for a large variety of continuous nonlinear systems. Our methodology resembles that of the applied computational tools used to solve control problems [13. Specifically, we consider a system of the form $\dot{\mathbf{x}}=\boldsymbol{\Phi}(\mathbf{x}, \mathbf{u}),(\Sigma)$, where the components of $\boldsymbol{\Phi}, \Phi_{i}$, are multivariable polynomials. According to the theory, if the linearization of $(\Sigma)$ at the origin is asymptotically controllable (i.e., all the uncontrollable eigenvalues have negative real parts), then the nonlinear system is locally asymptotically stabilizable [14]. This means that we can find a feedback-law that makes the closed-loop system asymptotically stable to the origin. The aim of this paper is to calculate those feedback-laws computationally by means of certain symbolic algorithms. Concretely, we seek for nonlinear state-feedbacks of the form $\mathbf{u}=\mathbf{a}(\mathbf{x})$, where $\mathbf{a}(\mathbf{x})$ consists of multivariable polynomials, so that a proper Lyapunov function becomes negative. To solve the problem we develop the following algorithms:

The Formal Algorithm. This algorithm allows us to write a polynomial $p$ as follows:

$$
\begin{gathered}
p=c_{1}\left(W_{i, \sigma, \varphi}\right) x_{1}^{j_{1,1}} \cdot\left[W_{2,1,1} x_{1}+x_{2}\right]^{j_{2,1}} \cdot\left[W_{3,1,1} x_{1}+W_{3,2,1} x_{2}+x_{3}\right]^{j_{3,1}} \cdots \\
\cdots\left[W_{n, 1,1} x_{1}+W_{n, 2,1} x_{2}+\cdots+x_{n}\right]^{j_{n, 1}}+ \\
+c_{2}\left(W_{i, \sigma, \varphi}\right) x_{1}^{j_{1,2}} \cdot\left[W_{2,1,2} x_{1}+x_{2}\right]^{j_{2,2}} \cdot\left[W_{3,1,2} x_{1}+W_{3,2,2} x_{2}+x_{3}\right]^{j_{3,2}} \cdots \\
\cdots\left[W_{n, 1,2} x_{1}+W_{n, 2,2} x_{2}+\cdots+x_{n}\right]^{j_{n, 2}}+\cdots
\end{gathered}
$$




$$
\begin{gathered}
+c_{k}\left(W_{i, \sigma, \varphi}\right) x_{1}^{j_{1, k}} \cdot\left[W_{2,1, k} x_{1}+x_{2}\right]^{j_{2, k}} \cdot\left[W_{3,1, k} x_{1}+W_{3,2, k} x_{2}+x_{3}\right]^{j_{3, k}} \cdots \\
\cdots\left[W_{n, 1, k} x_{1}+W_{n, 2, k} x_{2}+\cdots+x_{n}\right]^{j_{n, k}}+R_{\mathcal{W}}\left(x_{1}\right)
\end{gathered}
$$

where the exponents $j_{a, b}$ are specific positive whole numbers, the quantities $W_{i, \sigma, \varphi}$ are undetermined parameters that can take certain values, $c_{j}\left(W_{i, \sigma, \phi}\right)$ the coefficients depending on the parameters $W_{i, \sigma, \phi}$ and $R_{\mathcal{W}}\left(x_{1}\right)$ a polynomial of the single variable $x_{1}$, called the remainder. Equation (11) is called the Formal-Linear-LikeFactorization of $p$ and appeared firstly in [15. The essential tool of this methodology is a continuous reduction of $p$, by means of an Euclidean division. This makes our method similar to others [9], [1]. Yet in our methodology we only deal with a specific polynomial, in addition to the Gröbner basis that works with a polynomial ideal.

The POS-Algorithm. This algorithm checks the positivity of a multivariable polynomial $p$. This task can be achieved by giving to $W_{i, \sigma, \phi}$ values such that all the non-square terms to be eliminated and the square terms have positive coefficients. A lot of work has been done in this direction and some of the results can be found in the following [1, [3], [12, to mention but a few. In recent years there has been a strong algorithmic approach to the whole issue, as can be seen, for instance, in [4], 6], [8]. A number of those algorithms dealt with the problem of the existence of a sum of squares decomposition [2, [7, [10]. That is when we are able to write a polynomial $p$ as $p=\sum_{i} f_{i}^{2}\left(x_{1}, x_{2}, \ldots, x_{n}\right)$. In our sum of squares, $p=$ $\sum_{i} f_{i}^{2}\left(x_{1}, x_{2}, \ldots, x_{n}\right)$, the quantities $f_{i}\left(x_{1}, x_{2}, \ldots, x_{n}\right)$ are " linear " polynomials of the variables $x_{1}, x_{2}, \ldots, x_{n}$, while in other approaches [10], $f_{i}\left(x_{1}, x_{2}, \ldots, x_{n}\right)$ can be arbitrary. Moreover, our methodology provides not a specific sum of squares but a whole class, depending on the various proper values of the parameters $W_{i, \sigma, \varphi}$. The Feedback-GAS-Algorithm. This algorithm accepts as input the polynomials $\Phi_{i}$ and a Lyapunov Function $L$, and calculates the feedback connection $\mathbf{u}=\mathbf{a}(\mathbf{x})$. To achieve this we assume that $\mathbf{a}(\mathbf{x})$ consist of a multivariable polynomial with parametric coefficients (we denote them by $A_{i_{1}, i_{2}, \ldots, i_{n}}^{(j)}$ ). Our aim is to determine those values of the parameters $W_{i, \sigma, \varphi}$ and $A_{i_{1}, i_{2}, \ldots, i_{n}}^{(j)}$ that make the Lyapunov function negative along the trajectories of the closed-loop system. This guarantees the stability of the origin. The main merits of our method are the following:

1) It constitutes a pragmatic computational method. Indeed, the feedback-laws are derived from symbolic computational algorithms. Appropriate software has been created for this purpose, and all the examples presented in the current paper have been studied with the aid of this software.

2) It provides us with a whole class of admissible controllers, as opposed to only a single one.

3) It works even when the linearization of the nonlinear system has an uncontrollable eigenvalue with zero real part, [14]. 
4) Since the Lyapunov function, upon construction, is negative for every point, the stability is global.

The paper is divided into two parts. The first part is devoted to the presentation of the algorithms used throughout the paper. The second part is devoted to the specific control application. $\mathbf{R}$ and $\mathbf{Z}^{+}$will denote the sets of real numbers and positive integers correspondingly.

\section{The Algebraic Background}

Let $\mathbf{R}$ be the set of real numbers and $x_{1}, x_{2}, \ldots, x_{n} n$-variables. An expression of the form $p=\sum_{\lambda=1}^{\varphi} c_{\lambda} x_{1}^{a_{1, \lambda}} x_{2}^{a_{2, \lambda}} \cdots x_{n}^{a_{n, \lambda}}$, where $c_{\lambda} \in \mathbf{R}$ and some of the exponents $a_{i, j} \in \mathbf{Z}^{+}$are not equal to zero, is called a polynomial in $x_{1}, x_{2}, \ldots, x_{n}$ with real coefficients or, for short, a real polynomial. The set of all real polynomials in $x_{1}, x_{2}, \ldots, x_{n}$ is denoted by $\mathbf{R}\left[x_{1}, x_{2}, \ldots, x_{n}\right]$. An element $x_{1}^{a_{1, \lambda}} x_{2}^{a_{2, \lambda}} \cdots x_{n}^{a_{n, \lambda}}$ is called a monomial and an element $c_{\lambda} x_{1}^{a_{1, \lambda}} x_{2}^{a_{2, \lambda}} \cdots x_{n}^{a_{n, \lambda}}$ is called a term. Let $\phi_{n, \lambda}=x_{1}^{a_{1, \lambda}} x_{2}^{a_{2, \lambda}} \cdots x_{n}^{a_{n, \lambda}}$ and $\phi_{m, \mu}=x_{1}^{a_{1, \mu}} x_{2}^{a_{2, \mu}} \cdots x_{m}^{a_{m, \mu}}$ be two monomials. We defined the lexicographical order among monomials [9], as follows: we say that $\phi_{n, \lambda}$ is ordered less than $\phi_{m, \mu}$, denoted by $\phi_{n, \lambda} \prec \phi_{m, \mu}$, if either $n<m$ or $n=m$ and $a_{n, \lambda}<a_{m, \mu}$. In other words, the monomials are ordered as follows: $x_{1} \prec \cdots \prec x_{1}^{7} \prec$ $\cdots \prec x_{1} x_{2} \prec \cdots \prec x_{1} x_{2}^{8} \prec \cdots \prec x_{1} x_{2} x_{3} \prec \cdots$ Let $p$ be a given polynomial. Ordered lexicographically, the term that corresponds to the maximum monomial is called the maximum term denoted by maxterm $(p)$. Throughout the paper, a variable $W_{i, j, k}$ taking values in $\mathbf{R}$ is called an undetermined parameter. The set of undetermined parameters is denoted by $\mathcal{W}$.

Let $p \in \mathbf{R}\left[\mathrm{x}_{1}, \mathrm{x}_{2}, \ldots, \mathrm{x}_{\mathrm{n}}\right]$ be a polynomial with $n$-variables and $\mathcal{W}=\left\{W_{i, \sigma, \varphi}\right\}$ a set of undetermined parameters, taking values in $\mathbf{R}$. A Formal-Linear-LikeFactorization of $p$ is an expression of the form:

$$
\begin{gathered}
p=\sum_{\mu=1}^{k} c_{\mu}\left(W_{i, \sigma, \varphi}\right) x_{1}^{j_{1, \mu}} \cdot\left[W_{2,1, \mu} x_{1}+x_{2}\right]^{j_{2, \mu}} \cdot\left[W_{3,1, \mu} x_{1}+W_{3,2, \mu} x_{2}+x_{3}\right]^{j_{3, \mu}} \cdots \\
\cdots\left[W_{n, 1, \mu} x_{1}+W_{n, 2, \mu} x_{2}+\cdots+x_{n}\right]^{j_{n, \mu}}+R_{\mathcal{W}}\left(x_{1}\right)
\end{gathered}
$$

where the coefficients $c_{\mu}\left(W_{i, \sigma, \varphi}\right)$ are polynomial functions of the parameters $W_{i, \sigma, \varphi}$ and the remainder $R_{\mathcal{W}}\left(x_{1}\right)$ is a polynomial only of the single variable $x_{1}$, with coefficients depending on the parameters $W_{i, \sigma, \varphi}$, too. Some of the exponents $j_{1, \mu}, j_{2, \mu}, \ldots, j_{n, \mu} \in \mathbf{Z}^{+}$, may be equal to zero. The Formal-Linear-Like-Factorization of $p$ is denoted by FormalLF $[p]$. In a number of instances, a Formal-Linear-LikeFactorization of $p$, is written for short as Formal $L F[p]=\sum_{\mu=1}^{k} c_{\mu} L_{1, \mu}^{j_{1, \mu}} \cdot L_{2, \mu}^{j_{2, \mu}}$. $L_{3, \mu}^{j_{3, \mu}} \cdots L_{n, \mu}^{j_{n, \mu}}+R$, where $L_{\sigma, \mu}=W_{\sigma, 1, \mu} x_{1}+W_{\sigma, 2, \mu} x_{2}+\cdots+x_{\sigma}, \sigma=1, \ldots, n$ and $c_{\mu}, R$ are the abbreviations of $c_{\mu}\left(W_{i, \sigma, \varphi}\right)$ and $R_{\mathcal{W}}\left(x_{1}\right)$. 
Example 2.1 We have the polynomial $p=5 x_{1}-7 x_{1} x_{2}+11 x_{1} x_{3}$. The FormalLinear-Like-Factorization of $p$ is, $p=11 x_{1}\left(W_{3,1,1} x_{1}+W_{3,1,2} x_{2}+x_{3}\right)+(-7-$ $\left.11 W_{3,1,2}\right) x_{1}\left(W_{2,1,2} x_{1}+x_{2}\right)+5 x_{1}+\left(7 W_{2,1,2}-11 W_{3,1,1}+11 W_{2,1,2} W_{3,2,1}\right) x_{1}^{2}$.

The following theorem deals with the question of uniqueness of the Formal-LinearLike-Factorization.

Theorem 2.1 For a given polynomial $p \in \mathbf{R}\left[\mathrm{x}_{1}, \mathrm{x}_{2}, \ldots, \mathrm{x}_{\mathrm{n}}\right]$, the FormalLF $[p]$ is unique, under the assumption that the parameters $\mathcal{W}=\left\{W_{i, j, \varphi}\right\}$ are considered as constants.

Proof: If $p$ has only $x_{1}$-terms, the proof of the theorem is trivial, with $c_{\mu}=0$ and $R=p$. Let us suppose that $p$ has at least one term other than the $x_{1}$-terms. Let us further suppose that $\lambda x_{1}^{j_{1, h}} x_{2}^{j_{2, h}} \cdots x_{n}^{j_{n, h}}$ is its maximum term. This term appears also in the product: $c_{h} L_{1, h}^{j_{1, h}} L_{2, h}^{j_{2, h}} \cdots L_{n, h}^{j_{n, h}}$. By equating their coefficients, we calculate the quantity $c_{h}$, uniquely, actually $c_{h}=\lambda$. Repeating the same procedure for the term with the next higher order, we find an expression for the "next" coefficient $c_{h-1}$. Since this expression is a function of $c_{h}$ and some of the parameters $W_{i, \sigma, \varphi}$ are considered as constants, we conclude that $c_{h-1}$ is also defined uniquely. By induction, we finally get coefficients $c_{\mu}, \mu=1, \ldots, k$, all of which are uniquely determined. The polynomial $R$ consists only of $x_{1}$-terms. These terms arise either from the polynomial $p$ or from the products $c_{\mu} L_{1, \mu}^{j_{1, \mu}} \cdot L_{2, \mu}^{j_{2, \mu}} \cdot L_{3, \mu}^{j_{3, \mu}} \cdots L_{n, \mu}^{j_{n, \mu}}$. The unique determination of the coefficients $c_{\mu}$ entails the uniqueness of $R$, and the theorem has been proved.

What is of interest is the issue of constructing the Formal-Linear-Like-Factorization of a given polynomial. This we can do through the algorithm we describe below. It is based on a methodology that is analogous to the usual Euclidean division among polynomials and can be implemented on a computer via proper software (MATHEMATICA, for instance).

\section{THE FORMAL ALGORITHM}

Input: A multivariable polynomial $p$, a set of undetermined parameters $\mathcal{W}=\left\{W_{i, \sigma, \varphi}\right\}$, taking values in $\mathbf{R}$.

Initial Conditions: $k=0$

Step 1: We set $k=k+1$.

Step 2: We find the maximum term of $p, \operatorname{maxterm}(p)=c_{k} x_{1}^{j_{1, k}} \cdots x_{n}^{j_{n, k}}$. The coefficient $c_{k}$, in the first iteration, is a constant number. Then, it depends on the set of parameters $\mathcal{W}$.

Step 3: We form the linear polynomials: $L_{1, k}=x_{1}, L_{2, k}=W_{2,1, k} x_{1}+x_{2}, \ldots, L_{n, k}=$ $W_{n, 1, k} x_{1}+W_{n, 2, k} x_{2}+W_{n, 3, k} x_{3}+\cdots+x_{n}$.

Step 4: We make the subtraction: $R_{k}=p-c_{k} L_{1, k}^{j_{1, k}} L_{2, k}^{j_{2, k}} \cdots L_{n, k}^{j_{n, k}}$. 
Step 5: IF $R_{k}$ depends only on the variable $x_{1}$ THEN set $R=R_{k}$ and go to the output ELSE put $p=R_{k}$ and go to step 1.

Output: The quantities $R, c_{\mu}, L_{i, \mu}, j_{i, \mu}, \mu=1, \ldots, k, i=1, \ldots, n$

The following theorem proves the finiteness and the efficiency of the algorithm.

Theorem 2.2 The Formal Algorithm terminates after a finite number of steps. If $R, c_{\mu}, L_{i, \mu}, j_{i, \mu}, \mu=1, \ldots, k, i=1, \ldots, n$ are its outputs, then:

$$
\text { Formal } L F[p]=\sum_{\mu=1}^{k} c_{\mu} L_{1, \mu}^{j_{1, \mu}} \cdot L_{2, \mu}^{j_{2, \mu}} \cdot L_{3, \mu}^{j_{3, \mu}} \cdots L_{n, \mu}^{j_{n, \mu}}+R
$$

Proof: Let $p$ be a multivariable polynomial and $z=\gamma x_{1}^{a_{1}} x_{2}^{a_{2}} \cdots x_{n}^{a_{n}}$ its maximum term. We follow the Formal Algorithm step by step. When $k=1$, step 2 will give $c_{1}=\gamma$ and $j_{1,1}=a_{1}, \ldots, j_{n, 1}=a_{n}$. Taking into consideration the above values and the construction of the linear polynomials $L_{1,1}, L_{2,1}, \ldots, L_{n, 1}$, step 4 will produce a new polynomial $R_{1}$, which will not contain the term $z$. Obviously, the maximum term of $R_{1}$, which is called $z_{1}$, will be ordered lower than $z, z_{1} \prec z$, with respect to the order raised earlier. By induction, we get for the maximum terms $z \succ z_{1} \succ z_{2} \succ z_{3} \succ \cdots$. This nest and the construction of the order will finally eliminate all but $x_{1}$-terms. This fact guarantees the termination of the algorithm. Substituting now reversely, we have successively $p=c_{1} L_{1,1}^{j_{1,1}} \cdots L_{n, 1}^{j_{n, 1}}+R_{1}, R_{1}=$ $c_{2} L_{1,2}^{j_{1,2}} \cdots L_{n, 2}^{j_{n, 2}}+R_{2}, \cdots, R_{k-1}=c_{k} L_{1, k}^{j_{1, k}} \cdots L_{n, k}^{j_{n, k}}+R$. Combining these we get: $p=\sum_{\mu=1}^{k} c_{\mu} L_{1, \mu}^{j_{1, \mu}} \cdots L_{n, \mu}^{j_{n, \mu}}+R$, which is the Formal-Linear-Like-Factorization upon request.

The following example exhibits the function of the algorithm.

Example 2.2 We have the previous polynomial $p=5 x_{1}-7 x_{1} x_{2}+11 x_{1} x_{3}$. We want to find a Formal-Linear-Like-Factorization of $p$. In order to clarify our ideas we shall follow the Formal-Algorithm in detail. First, maxterm $(p)=11 x_{1} x_{3}$, here $c_{1}=11$ and $j_{1,1}=1, j_{1,2}=0, j_{1,3}=1$. The linear polynomials $L_{i, 1}$ are $L_{1,1}=x_{1}, L_{2,1}=W_{2,1,1} x_{1}+x_{2}, L_{3,1}=W_{3,1,1} x_{1}+W_{3,1,2} x_{2}+x_{3}$. Then we get $R_{1}=p\left(x_{1}, x_{2}, x_{3}\right)-11 L_{1,1}^{1} \cdot L_{2,1}^{0} \cdot L_{3,1}^{1}=5 x_{1}-7 x_{1} x_{2}-11 x_{1}\left(W_{3,1,1} x_{1}+W_{3,1,2} x_{2}+x_{3}\right)=$ $5 x_{1}-11 W_{3,1,1} x_{1}^{2}+\left(-7-11 W_{3,1,2}\right) x_{1} x_{2}$. The new maxterm is $\left(-7-11 W_{3,1,2}\right) x_{1} x_{2}$, with $c_{2}=-7-11 W_{3,1,2}, j_{1,2}=1, j_{2,2}=1$. The polynomials $L_{i, 2}$ now become $L_{1,2}=x_{1}, L_{2,2}=W_{2,1,2} x_{1}+x_{2}$ and $R_{2}$ is, $R_{2}=R_{1}-\left(-7-11 W_{3,1,2}\right) x_{1}\left(W_{2,1,2} x_{1}+\right.$ $\left.x_{2}\right)=5 x_{1}+\left(7 W_{2,1,2}-11 W_{3,1,1}+11 W_{2,1,2} W_{3,2,1}\right) x_{1}^{2} . \quad R_{2}$ contains only $x_{1}$-terms and the algorithm terminates. Therefore, the Formal Linear-Like Factorization of $p$ is $p=11 x_{1}\left(W_{3,1,1} x_{1}+W_{3,1,2} x_{2}+x_{3}\right)+\left(-7-11 W_{3,1,2}\right) x_{1}\left(W_{2,1,2} x_{1}+x_{2}\right)+$ $+5 x_{1}+\left(7 W_{2,1,2}-11 W_{3,1,1}+11 W_{2,1,2} W_{3,2,1}\right) x_{1}^{2}$

We can take different expressions of a concrete polynomial $p$, by giving certain values to the parameters $W_{i, \sigma, \varphi}$. Such procedures are called evaluations of the 
Formal $L F[p]$. The most rigorous approach is the following: Let $\mathcal{W}=\left\{W_{i, \sigma, \varphi}\right\}$ be the set of the variables that appear in the Formal - Linear - Like - Factorization of a given polynomial $p$. By arranging the parameters in an increasing order we form the vector $\mathcal{W}=\left(W_{i_{k}, \sigma_{k}, \varphi_{k}}\right)_{k=1,2, \ldots, n}$. Let $\mathbf{r}=\left(a_{k}\right)_{k=1,2, \ldots, n}$ be a vector of real numbers which has the same length as the vector $\mathcal{W}$. We say that the parameters $\mathcal{W}$ follow the rules $\mathbf{r}$ and we write $\mathcal{W} \rightarrow \mathbf{r}$ if the following substitution is valid: $W_{i_{k}, \sigma_{k}, \varphi_{k}}=a_{k}, k=1,2, \ldots, n$. Let $M$ a set of rules, $M=\left\{\mathbf{r}_{1}, \mathbf{r}_{2}, \ldots, \mathbf{r}_{\lambda}\right\}$ then

$$
\text { FormalLF }\left.[p]\right|_{M}=\bigcup_{\nu=1}^{\lambda}\left\{\sum_{\mu=1}^{k} c_{k} L_{1, \mu}^{j_{1, \mu}} \cdots L_{n, \mu}^{j_{n, \mu}}+R \quad \text { with } \quad \mathcal{W} \rightarrow \mathbf{r}_{\nu} \in M\right\}
$$

The set of substitutions $M$, may be finite or infinite.

Example 2.3 We shall work with the polynomial $p$ that appears in the example (2.2). Let $M=\{(-2,1,-1)\}$. We determine the following order among the parameters $\left(W_{3,1,1}, W_{3,2,1}, W_{2,1,2}\right)$. Then $W_{3,1,1}=-2, W_{3,2,1}=1, W_{2,1,2}=-1$ and

FormalLF $\left.[p]\right|_{M}=11 x_{1}\left(-2 x_{1}+x_{2}+x_{3}\right)-18 x_{1}\left(-x_{1}+x_{2}\right)+5 x_{1}+4 x_{1}^{2}$. If $M=\{(\varphi, \varphi, \theta)\}, \varphi, \theta \in \mathbf{R}$ then we set $W_{3,1,1}=\varphi, W_{3,2,1}=\varphi, W_{2,1,2}=\theta$ and FormalLF $\left.[p]\right|_{M}=11 x_{1}\left(\varphi x_{1}+\varphi x_{2}+x_{3}\right)+(-7-11 \varphi) x_{1}\left(\theta x_{1}+x_{2}\right)+5 x_{1}+(7 \theta-$ $11 \varphi+11 \theta \varphi) x_{1}^{2}$. This is just a re-parameterization of the FormalLF $[p]$.

In this section we shall examine the positivity of multivariable polynomials via the Formal-Linear-Like-Factorizations developed above. This is a well-known issue and there are and other computational approaches - recently, for instance, in [10] - that are mainly based on algorithms that construct sums of squares. Our contribution essentially relies on the existence of parameters that provide us with a more flexible tool that is also suitable for solving the positivity problem, among others. A given polynomial $p \in \mathbf{R}\left[x_{1}, x_{2}, \ldots, x_{n}\right]$, is called positive, if $p \geq 0$, for every $\left(x_{1}, x_{2}, \ldots, x_{n}\right) \in \mathbf{R}^{n}$. Let $\mathcal{S}$ be the set of values of the parameters $W_{i, \sigma, \varphi}$, such that all but the square terms of $p$ are eliminated and the coefficients of the remaining terms are positive real numbers. Then, if $\mathcal{S} \neq \emptyset$ the polynomial is positive and FormalLF $\left.[p]\right|_{\mathcal{S}}$ ia a class of sum of squares of $p$. Here we need to make clear that all the calculations are symbolic and not numerical. This permits us to study not only specific polynomials with numeric coefficients, but also classes of polynomials with parametric coefficients. In any case, the construction of the set $\mathcal{S}$ can be carried out via the following algorithm:

\section{THE POS-ALGORITHM}

Input: A multivariable polynomial $p$, a set of undetermined parameters $\mathcal{W}=\left\{W_{i, \sigma, \varphi}\right\}$, taking values in $\mathbf{R}$.

Initial Conditions: $\mathcal{S}=\{\}, E=\{\}$.

Step 1: By means of the FORMAL ALGORITHM we get the quantities $R, c_{\mu}, L_{i, \mu}, j_{i, \mu}$, $\mu=1, \ldots, k, i=1, \ldots, n$. We denote the coefficients of the remainder $R$ by $c_{\mu}$, $\mu=k+1, \ldots, k+h, h$ the number of terms of $R$. 
Step 2: REPEAT FOR $\mu=1, \ldots, k+h$

IF some of the exponents $j_{i, \mu}, i=1, \ldots, n$ are odd numbers THEN $O=O \cup\left\{c_{\mu}\right\}$ ELSE $E=E \cup\left\{c_{\mu}\right\}$

\section{NEXT $\mu$}

Step 3: Find the values of the parameters $W_{i, j, \phi}$ that eliminate the " odd " coefficients and make the "even" coefficients positive. In other words, we construct the set $S=\left\{W_{i, j, \phi}=\lambda_{i, j, \phi} \in \mathbf{R}: c_{\mu}=0\right.$ and $c_{p} \geq 0$ with $c_{\mu} \in O$ and $\left.c_{p} \in E\right\}$.

Output: The set $\mathcal{S}$.

The proof of the following theorem is straightforward.

Theorem 2.3 Let $p \in \mathbf{R}\left[x_{1}, x_{2}, \ldots, x_{n}\right]$ be a given multivariable polynomial. If $\mathcal{S}$ is non-void, then $p$ is positive definite, and FormalLF $\left.[p]\right|_{\mathcal{S}}$ is a family of " sum of squares " expressions of $p$.

We would like to make the following remarks in connection with the above algorithm:

Remark 2.1 (i) We can modify the algorithm so that the whole procedure is executed " together" with the Formal Algorithm, as opposed to after it. This will allow for a quicker implementation of the method. (ii) Let us suppose that we have a Formal - Linear - Like - Factorization of a given polynomial p. The coefficients of the first terms with odd exponents contain a small number of parameters ( usually one or two). This means that they can be eliminated easily for some particular values of the $W$-parameters. By substituting those values into the other terms we decrease the number of parameters, thus simplifying the whole computational procedure significantly. Clearly, the method is not computationally complex and can be carried out normally. Certain algorithms can be used towards this direction, for instance the Stetter algorithn, to mention but a few. (iii) If the output of the POSAlGORITHM is $\mathcal{S}=\emptyset$, this does not mean that the polynomial $p$ is not positive or that another sum of squares does not exist. Nevertheless, our approach can be extended by using nonlinear " factors ", for instance: $\tilde{L}_{n, k}=W_{n, 1, k} x_{1}+W_{n, 2, k} x_{2}+$ $\cdots+W_{n,(1,2), k} x_{1} x_{2}+\cdots+W_{n,(n-1, n-1), k} x_{n-1}^{2}+x_{n}^{2}$. In this case we can obtain a further sums of squares that can successfully deal with cases in which the current method fails. This will be undertaken in a future study.

Example 2.4 Let us consider the polynomial $p=x^{2}-2 x y+6 y^{2}-4 y z+3 z^{2}$. Its Formal-Linear-Like-Factorization is: $3\left(z+x W_{3,1,1}+y W_{3,2,1}\right)^{2}+\left(y+x W_{2,1,2}\right)\left(-6 W_{3,2,1}-\right.$ 4) $\left(z+x W_{3,1,2}+y W_{3,2,2}\right)+x\left(6 W_{3,2,1} W_{2,1,2}+4 W_{2,1,2}-6 W_{3,1,1}\right)\left(z+x W_{3,1,3}+y W_{3,2,3}\right)+$ $\left(y+x W_{2,1,4}\right)^{2}\left(-3 W_{3,2,1}^{2}+6 W_{3,2,2} W_{3,2,1}+4 W_{3,2,2}+6\right)+x\left(y+x W_{2,1,5}\right)\left(6 W_{2,1,4} W_{3,2,1}^{2}-\right.$ $6 W_{3,1,1} W_{3,2,1}+6 W_{3,1,2} W_{3,2,1}+6 W_{2,1,2} W_{3,2,2} W_{3,2,1}-12 W_{2,1,4} W_{3,2,2} W_{3,2,1}-6 W_{2,1,2} W_{3,2,3} W_{3,2,1}$ $-12 W_{2,1,4}+4 W_{3,1,2}+4 W_{2,1,2} W_{3,2,2}-8 W_{2,1,4} W_{3,2,2}-4 W_{2,1,2} W_{3,2,3}+6 W_{3,1,1} W_{3,2,3}-$ 
$2)+R$ (We do not include the entire remainder because of its size). In order to eliminate the first non-square term to appear, we set $W_{3,2,1}=-\frac{2}{3}$. This transforms the factorization as follows: $3\left(-\frac{2 y}{3}+z+x W_{3,1,1}\right)^{2}-6 x W_{3,1,1}\left(z+x W_{3,1,3}+\right.$ $\left.y W_{3,2,3}\right)+\frac{14}{3}\left(y+x W_{2,1,4}\right)^{2}-\frac{2}{3} x\left(y+x W_{2,1,5}\right)\left(14 W_{2,1,4}-6 W_{3,1,1}-9 W_{3,1,1} W_{3,2,3}+3\right)$ $+R^{\prime}$. The values $W_{3,1,1}=0$ and $W_{2,1,4}=-\frac{3}{14}$ eliminate the other non-square terms and the factorization becomes $p=3\left(-\frac{2 y}{3}+z\right)^{2}+\frac{14}{3}\left(-\frac{3 x}{14}+y\right)^{2}+\frac{11}{14} x^{2}$. Thus, the set $\mathcal{S}$ upon request is $\mathcal{S}=\left\{W_{3,2,1}=-\frac{2}{3}, W_{3,1,1}=0, W_{2,1,4}=-\frac{3}{14}\right.$ all the other parameters $W_{i, j, \varphi}$ are free $\}$. The above" sum of squares "expression of $p$ guarantees that $p$ is positive.

\section{The Feedback Asymptotic Stabilization}

We are now in a position to apply the entire concept that was raised in earlier sections to the problem of feedback asymptotically stabilizing a nonlinear system at a given equilibrium point. This is a well-known topic that has been discussed extensively in the literature [5], 14]. In this paper we adopt a computational approach. Specifically, let us have the continuous nonlinear system: $\dot{\mathbf{x}}=\boldsymbol{\Phi}(\mathbf{x}, \mathbf{u})$, where $\mathbf{x}=\left(x_{1}, x_{2}, \ldots, x_{n}\right)^{T}$ is the state vector, $\mathbf{u}=\left(u_{1}, u_{2}, \ldots, u_{m}\right)^{T}$ the input vector and $\mathbf{\Phi}=\left[\Phi_{1}(\mathbf{x}, \mathbf{u}), \ldots, \Phi_{n}(\mathbf{x}, \mathbf{u})\right]^{T}$, where $\Phi_{i}(\mathbf{x}, \mathbf{u}), i=1, \ldots, n$ are multivariable polynomials of $\left(x_{1}, \ldots, x_{n}\right)$ and $\left(u_{1}, \ldots, u_{m}\right)$ without free terms. Obviously $\left(\mathbf{x}^{0}, \mathbf{u}^{0}\right)=$ $(\mathbf{0}, \mathbf{0})$ is an equilibrium point. Let us denote by $(A, B)$ the linearization pair of this nonlinear system "around" the origin, (i.e. $A=\frac{\partial \Phi}{\partial \mathbf{x}}(\mathbf{0}, \mathbf{0}), B=\frac{\partial \Phi}{\partial \mathbf{u}}(\mathbf{0}, \mathbf{0})$ ). As is already known, 14], if the linear system $(A, B)$ is asymptotically controllable (which we can check by testing the sign of the real parts of the uncontrollable eigenvalues. If all of these are negative, then the system is asymptotically controllable), then the corresponding nonlinear system is locally asymptotically stable at the origin. This means that we can find a matrix $F$ such that the feedback-law constructs a system $\dot{\mathbf{x}}=\mathbf{\Phi}(\mathbf{x}, F(\mathbf{x}))$ which is locally asymptotically stable at the point $(\mathbf{0}, \mathbf{0})$. The main concern of this paper is to calculate this quantity $F$ computationally, but with the following alterations:

1) $F$ does not need to be linear (a matrix) but may also be nonlinear (a polynomial function). Actually, the problem under examination is that of finding a state feedback of the form $\mathbf{u}=\mathbf{a}(\mathbf{x})$, with $\mathbf{a}(\mathbf{x})=\left[a_{1}(\mathbf{x}), \ldots, a_{m}(\mathbf{x})\right]^{T}$ and $a_{i}(\mathbf{x}), i=1, \ldots, m$ multivariable polynomials too, where the corresponding closedloop system $\dot{\mathbf{x}}=\boldsymbol{\Phi}(\mathbf{x}, \mathbf{a}(\mathbf{x})))$ has a global asymptotically stable equilibrium at $\left(\mathbf{x}^{0}, \mathbf{u}^{0}\right)=(\mathbf{0}, \mathbf{0})$.

2) Some of the uncontrollable eigenvalues of $(A, B)$ can have zero real parts.

3) The stability is not local but global.

At this point we introduce the algorithm in order to deal with the feedback asymptotic stabilization problem.

THE FEEDBACK-GAS ALGORITHM 
Input: The polynomials $\Phi_{i}(\mathbf{x}, \mathbf{u})$, a polynomial Lyapunov function $L$, the degree of the feedback law upon request.

Step 1: We define the feedback law $\mathbf{u}=\mathbf{a}(\mathbf{x}), \mathbf{a}(\mathbf{x})=\left[a_{1}(\mathbf{x}), a_{2}(\mathbf{x}), \ldots, a_{n}(\mathbf{x})\right]$, with

$$
\begin{gathered}
a_{j}(\mathbf{x})=\sum_{i_{1}=1}^{n} A_{i_{1}}^{(j)} x_{i_{1}}+\sum_{\left(i_{1}, i_{2}\right)=(1,1)}^{(n, n)} A_{\left(i_{1}, i_{2}\right)}^{(j)} x_{i_{1}} x_{i_{2}}+ \\
\cdots+\sum_{\left(i_{1}, i_{2}, \ldots, i_{k}\right)=(1,1, \ldots, 1)}^{(n, n, \ldots, n)} A_{\left(i_{1}, i_{2}, \ldots, i_{n}\right)}^{(j)} x_{i_{1}} x_{i_{2}} \cdots x_{i_{n}}
\end{gathered}
$$

and $A_{\left(i_{1}, i_{2}, \ldots, i_{n}\right)}^{(j)}$ unknown parameters taking values in $\mathbf{R}$.

Step 2: We define the quantity:

$$
V=-\frac{\partial L}{\partial \mathbf{x}}\left(\Phi_{1}(\mathbf{x}, \mathbf{a}(\mathbf{x})), \Phi_{2}\left(\mathbf{x}, \ldots, \Phi_{n}(\mathbf{x}, \mathbf{a}(\mathbf{x}))\right.\right.
$$

Step 3: By means of the POS-ALGORITHM we construct the set $\mathcal{V}$, consisting of those values of the parameters for which $V$ is positive.

Output: The set $\mathcal{V}$.

Theorem 3.1 Let us have the nonlinear continuous system: $\dot{\mathbf{x}}=\mathbf{\Phi}(\mathbf{x}, \mathbf{u})$. Let $\mathcal{V}$ be the output of the FEEDBACK-GAS-Algorithm. If $\mathcal{V} \neq \emptyset$ then the set of feedback laws $\mathbf{u}=\left.\mathbf{a}(\mathbf{x})\right|_{\mathcal{V}}$ make the origin globally asymptotically stable.

Proof: The positive definiteness of the quantity $V$ guarantees that the Lyapunov function $L$ decreases along the trajectories of the closed-loop system $\mathbf{\Phi}(\mathbf{x}, \mathbf{a}(\mathbf{x}))$ and, therefore, the origin is asymptotically stable. Given that this is the case for every point, the origin is globally asymptotically stable.

Example 3.1 The angular momentum of a rigid body controlled by two independent torques can be described, following some simplification, through the equations: $\dot{\mathbf{x}}=\mathbf{\Phi}(\mathbf{x}, \mathbf{u})=\left[\begin{array}{l}\Phi_{1}(\mathbf{x}, \mathbf{u}) \\ \Phi_{2}(\mathbf{x}, \mathbf{u}) \\ \Phi_{3}(\mathbf{x}, \mathbf{u})\end{array}\right]$, with $\mathbf{x}=\left[x_{1}, x_{2}, x_{3}\right], \mathbf{u}=\left[u_{1}(t), u_{2}(t)\right]$ and $\Phi_{1}=a_{1} x_{2} x_{3}+u_{1}, \Phi_{2}=a_{2} x_{1} x_{3}+u_{2}, \Phi=a_{3} x_{1} x_{2}$. The quantities $a_{1}, a_{2}$ and $a_{3}$ are certain constants and $a_{3} \neq 0$, ([14], page 176). It can be proved that this system can be globally stabilized about $\mathbf{x}=\mathbf{0}$ and $\mathbf{u}=\mathbf{0} . \quad$ A specific feedback can be constructed by following certain methods [14]. Following the steps of the Feedback-GAS-Algorithm we select a Lyapunov function of the form $L=x_{1}^{2}+x_{2}^{2}+x_{3}^{2}$, and a pair of feedback-laws of the forms: $u_{1}=A_{1} x_{1}+B_{1} x_{2}+$ $\Gamma_{1} x_{3}+\Delta_{1} x_{2} x_{3}, u_{2}=A_{2} x_{1}+B_{2} x_{2}+\Gamma_{2} x_{3}+\Delta_{2} x_{1} x_{3}$. Then, we define the quantity: 
$V=-\Phi_{1}\left(x_{1}, x_{2}, x_{3}, A_{1} x_{1}+B_{1} x_{2}+\Gamma_{1} x_{3}+\Delta_{1} x_{2} x_{3}\right) x_{1}-\Phi_{2}\left(x_{1}, x_{2}, x_{3}, A_{2} x_{1}+B_{2} x_{2}+\right.$ $\left.\Gamma_{2} x_{3}+\Delta_{2} x_{2} x_{3}\right) x_{2}-\Phi_{3}\left(x_{1}, x_{2}, x_{3}\right) x_{3}$. The Formal Algorithm will give the following Formal-Linear-Like-Factorization of $V$ :

$$
\begin{gathered}
\left(a_{1}+a_{2}+a_{3}+\Delta_{1}+\Delta_{2}\right)\left[W_{2,1,1}\left(x_{3}+x_{1} W_{3,1,3}+x_{2} W_{3,2,3}\right) x_{1}^{2}\right. \\
+\left(x_{2}+x_{1} W_{2,1,7}\right)\left(W_{3,1,1}+W_{2,1,1} W_{3,2,1}-2 W_{2,1,5} W_{3,2,1}-W_{2,1,1} W_{3,2,3}\right) x_{1}^{2}+ \\
\left.\left(x_{2}+x_{1} W_{2,1,5}\right)^{2}\left(W_{3,2,1} x_{1}-x_{2}-x_{1} W_{2,1,1}\right)\left(x_{3}+x_{1} W_{3,1,1}+x_{2} W_{3,2,1}\right) x_{1}\right]+ \\
\left(\Gamma_{2} W_{2,1,2}-\Gamma_{1}\right)\left(x_{3}+x_{1} W_{3,1,4}+x_{2} W_{3,2,4}\right) x_{1}+\left(x_{2}+x_{1} W_{2,1,8}\right) \\
\left(-A_{2}-B_{1}+2 B_{2} W_{2,1,6}+\Gamma_{2} W_{3,1,2}+\Gamma_{2} W_{2,1,2} W_{3,2,2}-2 \Gamma_{2} W_{2,1,6} W_{3,2,2}+\Gamma_{1} W_{3,2,4}-\right. \\
\left.\Gamma_{2} W_{2,1,2} W_{3,2,4}\right) x_{1}+\left(x_{2}+x_{1} W_{2,1,6}\right)^{2}\left(\Gamma_{2} W_{3,2,2}-B_{2}\right)- \\
\Gamma_{2}\left(x_{2}+x_{1} W_{2,1,2}\right)\left(x_{3}+x_{1} W_{3,1,2}+x_{2} W_{3,2,2}\right)+R
\end{gathered}
$$

where $R$ is the remainder. The values of the parameters that eliminate the nonsquares terms of the above expressions are $W_{2,1,1}=0, W_{3,2,1}=0, W_{3,1,1}=0$, $W_{2,1,4}=\frac{A_{2}+B_{1}}{2 B_{2}}, \Gamma_{1}=0, \Gamma_{2}=0, \Delta_{1}=-\Delta_{2}-a_{1}-a_{2}-a_{3}$ and $A_{1}, A_{2}, B_{1}, B_{2}, \Delta_{2}$, arbitrary. For those specific values the FormalLF[V] becomes: $\frac{\left(A_{2}^{2}+2 B_{1} A_{2}+B_{1}^{2}-4 A_{1} B_{2}\right) x_{1}^{2}}{4 B_{2}}$ $-B_{2}\left(x_{2}+\frac{A_{2}+B_{1}}{2 B_{2}} x_{1}\right)^{2}$. In order to get positive coefficients we further demand $B_{2}<0$ and $A_{1}<\frac{A_{2}^{2}+2 B_{1} A_{2}+B_{1}^{2}}{4 B_{2}}$. These conditions mean that the Lyapunov function decreases along the trajectories of the system, which guarantees the asymptotic stability of the origin. The family of feedback laws is given by the relations $u_{1}=A_{1} x_{1}+B_{1} x_{2}+\Delta_{1} x_{1} x_{2}, u_{2}=A_{2} x_{1}+B_{2} x_{2}+\Delta_{2} x_{1} x_{3}$ with $B_{2}<0$, $A_{1}<\frac{A_{2}^{2}+2 B_{1} A_{2}+B_{1}^{2}}{4 B_{2}}, \Delta_{1}=\Delta_{2}-a_{1}-a_{2}-a_{3}$ and $A_{2}, B_{1}, \Delta_{2}$ arbitrary. We can also obtain other classes of feedback, by choosing other expressions for the quantities $u_{1}, u_{2}$ or other Lyapunov function.

Example 3.2 Let us suppose that we have the nonlinear system: $\dot{\mathbf{x}}=\mathbf{\Phi}(\mathbf{x}, \mathbf{u})=$ $\left[\begin{array}{l}\Phi_{1}(\mathbf{x}, \mathbf{u}) \\ \Phi_{2}(\mathbf{x}, \mathbf{u})\end{array}\right]$ with $\Phi_{1}=4 x+8 y-\frac{11}{16} x^{3}+5 y u_{1}-\frac{5}{2} u_{1} u_{2}-4 u_{2}+5 x u_{2}, \Phi_{2}=-\frac{55}{8} x^{2}-$ $3 y^{3}-\frac{1}{2} u_{2}^{2} x$, where $\mathbf{x}=[x(t), y(t)]^{T}$ is the state of the system, consisting of two functions and $\mathbf{u}=\left[u_{1}(t), u_{2}(t)\right]$ the input vector. We can easily check that the origin is an unstable equilibrium point for the system. Furthermore, the linearization of this system is not asymptotically controllable, since the polynomial $\chi_{u}$ has a root with zero real part (see [14] for details). Following the steps of the Feedback-GAS Algorithm, we choose a pair of feedback laws of the form: $u_{1}=A_{1} x+B_{1} y+\Gamma_{1} x y$, $u_{2}=A_{2} x+B_{2} y+\Gamma_{2} x y$. Then, we define the quantity $V=-\Phi_{1}\left(x, y, A_{1} x+B_{1} y+\right.$ $\left.\Gamma_{1} x y, A_{2} x+B_{2} y+\Gamma_{2} x y\right) x-\Phi_{2}\left(x, y, A_{1} x+B_{1} y+\Gamma_{1} x y, A_{2} x+B_{2} y+\Gamma_{2} x y\right) y$. The Formal Algorithm will give the following Formal-Linear-Like-Factorization of $V$ :

$$
\text { FormalLF }[V]=-\frac{1}{8} \Gamma_{2}^{2} W_{2,1,2}^{3} x^{6}-\frac{3}{2} \Gamma_{2}^{2} W_{2,1,2}\left(y+\frac{1}{2} x W_{2,1,2}\right)^{2} x^{4}+
$$




$$
\begin{gathered}
+\frac{-4 \Gamma_{2} A_{2}^{2}-20 \Gamma_{1} \Gamma_{2} A_{2}-25 \Gamma_{1}^{2} \Gamma_{2}}{12 B_{2}}\left(y+x W_{2,1,10}\right) x^{4}+\frac{1}{2} \Gamma_{2}^{2}\left(y+x W_{2,1,2}\right)^{3} x^{3}+ \\
+\frac{1}{32}\left(-B_{2}^{4}+32 A_{2} B_{2}+80 \Gamma_{1} B_{2}-160 \Gamma_{1}+80 \Gamma_{2} B_{1}\right) . \\
\cdot\left(y-x \frac{B_{2}^{6}-576 A_{2}^{2}-2880 A_{2} \Gamma_{1}-2880 A_{1} \Gamma_{2}+5760 \Gamma_{2}}{72\left(-B_{2}^{4}+32 A_{2} B_{2}+80 \Gamma_{1} B_{2}-160 \Gamma_{1}+80 \Gamma_{2} B_{1}\right)}\right)^{2} x^{2}+ \\
+\Gamma_{2}\left(y-x \frac{\left(-2 A_{2}-5 \Gamma_{1}\right)}{6 B_{2}}\right)^{3} B_{2} x^{2}+\frac{5}{2}\left(B_{1} B_{2}-2 B_{1}\right) \cdot \\
\cdot\left(y-x \frac{\left(-20 B_{2} A_{1}+40 A_{1}-32 \Gamma_{2}-20 A_{2} B_{1}+40 B_{2}-55\right)}{40 B_{1}\left(B_{2}-2\right)}\right)^{2} x+ \\
+4\left(B_{2}-2\right)\left(y+x W_{2,1,13}\right) x+3\left(x \frac{B_{2}^{2}}{24}+y\right)^{4}+R
\end{gathered}
$$

where $R$ is the remainder (not included because of its size). The values of the parameters that eliminate the non square terms of the above expression and make the coefficients of the remaining terms positive, are $\mathcal{V}=\left\{A_{1}=2, A_{2}=\frac{5}{4 B_{1}}\right.$, $B_{2}=2, \Gamma_{2}=0$, with $0<B_{1}<\frac{5}{4}$ and $\frac{16 B_{1}^{3}-225 B_{1}-32 \sqrt{111} \sqrt{5 B_{1}^{5}-B_{1}^{6}}}{900 B_{1}^{2}}<\Gamma_{1}<$ $\left.\frac{16 B_{1}^{3}-225 B_{1}+32 \sqrt{111} \sqrt{5 B_{1}^{5}-B_{1}^{6}}}{900 B_{1}^{2}}\right\}$ and $W_{i, j, \sigma}$ arbitrary. By taking, for instance, $B_{1}=$ $1, \Gamma_{1}=\frac{1}{2}$ the FormalLF $[V]$ becomes:

$$
\text { FormalLF }\left.[V]\right|_{\mathcal{V}}=\frac{20375}{663552} x^{4}+2\left(\frac{659}{1152} x+y\right)^{2} x^{2}+x^{2}+3\left(\frac{x}{6}+y\right)^{4}
$$

which means that the Lyapunov function decreases along the trajectories of the system. This guarantees the asymptotic stability of the origin. The family of the feedback laws is given by the relations $u_{1}=x+B_{1} y+\Gamma_{1} x y, u_{2}=\frac{5}{4 B_{1}} x+2 y$, with $0<B_{1}<\frac{5}{4}$, and $\frac{16 B_{1}^{3}-225 B_{1}-32 \sqrt{111} \sqrt{5 B_{1}^{5}-B_{1}^{6}}}{900 B_{1}^{2}}<\Gamma_{1}<\frac{16 B_{1}^{3}-225 B_{1}+32 \sqrt{111} \sqrt{5 B_{1}^{5}-B_{1}^{6}}}{900 B_{1}^{2}}$. It should be noted that the values 0 and $\frac{5}{4}$ for the parameter $B_{1}$ are a kind of bifurcation point for the feedback expression, above or below which the asymptotic stabilization is not achieved.

\section{References}

[1] J.B.Lasserre" Global optimization with polynomials and the problem of moments. " SIAM Journal on Optimization, Vol. 11, N0. 3, pp. 796-817, 2001.

[2] M.D.Choi and T. Y. Lam, and B. Reznick " Sums of squares of real polynomials. " Proceedings of Symposia in Pure Mathematics, 58 (2): 103-126, 1995. 
[3] M.A.Hasan and A.A.Hasan " A procedure for the positive definiteness of forms of even order. " IEEE Transactions on Automatic Control, 41(4): 615-617, 1996.

[4] D.S.Hochbaum, editor. " Approximation algorithms for NP-hard problems. " PWS Publishing Company, 1997.

[5] A. Isidori. " Nonlinear Control Systems. " Springer - Verlag, Berlin, third edition, 1997.

[6] B.Mishra." Algorithmic Algebra. " Springer-Verlag, 1993.

[7] V.Powers and T.Wörmann. " An algorithm for sums of squares of real polynomials." Journal of Pure and Applied Algebra,127, 99-104,1998.

[8] L.Vandenberghe and S.Boyd. " Semidefinite Programming ". SIAM Review, 38(1), 49-95, March 1996.

[9] D. Cox, J. Little, D. O'Shea. (1997). "Ideals, Varieties and Algorithms". Springer-Verlag, New York.

[10] P.A.Parrilo (2000) " Structured semidefinite programs and semialgebraic geometry methods in robustness and optimization ". PhD thesis, California Institute of Technology.

[11] P.A.Parrilo (2002) " An explicit construction of distinguished representations of polynomials nonnegative over finite sets ". IFA Technical Report AUT0202 .

[12] A.Prestel and C.N.Delzell, (2001)" Positive polynomials: from Hilbert's 17th problem to real algebra. " Springer Monographs in Mathematics. Springer, New York.

[13] N.Munro (editor), (1999) " Symbolic Methods in control System Analysis and Design." IEE Control Engineering Series, 56.

[14] E.Sontag (1997) " Mathematical Control Theory ", Texts in Applied Mathematics, Springer, New York.

[15] S.Kotsios (2003) "The problem of positive defineteness through a formal factorization of polynomials. " HERCMA - Congress 2003, ATHENS. 